\title{
Nanoparticle imaging could guide brain tumour surgery
}

A team of US researchers has developed a gold-silica nanoparticle that could be used for molecular imaging to guide surgery for brain tumours. The particles showed promise in mouse models of glioblastoma, the most aggressive form of brain cancer. "We chose to focus on the brain because it is very important to spare normal brain tissue in glioblastoma surgery," explains Sam Gambhir, who led the study.

To generate a model of glioblastoma, the researchers implanted human glioblastoma cells into the striata of mice. Nanoparticles were then administered by injection into the tail vein. Gambhir and colleagues hypothesized that the nanoparticles would only enter the brain at regions of tumourdriven damage to the blood-brain barrier, enabling targeting to the tumour site.

The 120-nm particles were coated with gadolinium $(\mathrm{Gd})$ to enable imaging with MRI. In addition, the core, being made of gold, is well-suited to a new technique called photoacoustic imaging, whereby light energy is used to heat the imaging agent, which then emits ultrasound waves. Moreover, between the gold core and the Gd coating was a Raman-active layer, which emits low levels of light at signature frequences, allowing high-resolution visualization of the particles using Raman imaging.

Gambhir and colleagues found good colocalization of tumour images acquired using all three imaging techniques through the intact skulls of live mice. Histological analysis confirmed that the nanoparticles accumulated at the glioblastoma site. The imaging signals remained high for $24 \mathrm{~h}$ after nanoparticle injection, in contrast to standard Gd-based agents, which undergo rapid washout.

The team used the nanoparticle signals to guide brain tumour resection in the mice. MRI scans, which delineate the gross structure of the tumour, were used to plan surgery, while photoacoustic imaging provided real-time information on tumour location during surgery. Finally, small foci of Raman signals were detected after the tumour seemed to be completely removed,

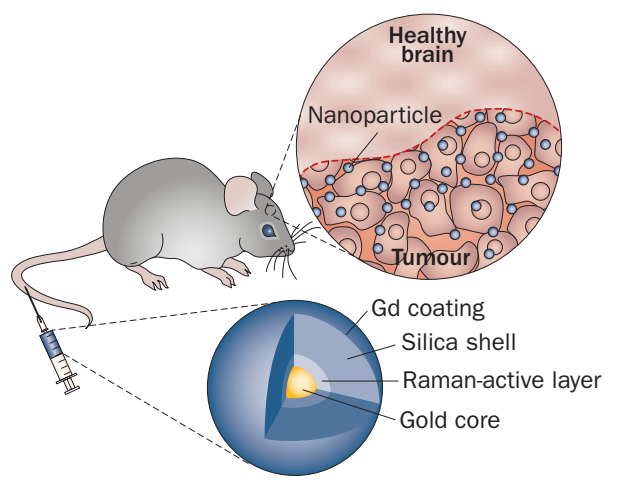

Permission obtained from Macmillan Publishers Ltd $\odot$ Kircher, M. F. et al. Nat. Med. 18, 829-834 (2012).

and were confirmed histologically as being tumour cells that would otherwise have been overlooked. "We are now working to develop nanoparticles with better targeting and signalling capacity," says Gambhir.

Katie Kingwell

Original article Kircher, M. F. et al. A brain tumor molecular imaging strategy using a new triple-modality MRI-photoacoustic-Raman nanoparticle. Nat. Med. 18, 829-834 (2012) 